\title{
Capacité germinative des graines et aptitude au bouturage des tiges, des racines tubéreuses de l'espèce Ampelocissus multistriata(Baker) Planch.
}

\author{
Mberdoum Memti NGUINAMBAYE ${ }^{*}$, Ngam-Asra NADJIOROUM ${ }^{2}$ et \\ Otchom Boy BRAHIM ${ }^{3}$
}

\author{
${ }^{1}$ Département de Biologie, Université de N’Djamena, Faculté des Sciences Exactes et Appliquées, Laboratoire \\ de Botanique systématique et d'Ecologie Végétale BP 1027 N'Djamena, Tchad. \\ ${ }^{2}$ Département de Biomédical, Université de Ndjamena, Faculté des Sciences de la Santé Humaine. BP 1117 \\ N'Djamena, Tchad. \\ ${ }^{3}$ Recteur de l'Université de Toumaï-BP 1764 N'Djamena, Tchad. \\ *Auteur correspondant ; E-mail :memti2020@gmail.com /memti2012@hotmail.fr
}

\author{
Received: 22-09-2020 Accepted: 15-08-2021 $\quad$ Published: 31-08-2021
}

\section{RESUME}

Cette étude s'inscrit dans le contexte de la valorisation des espèces végétales spontanées ayant des valeurs nutritives et économiques afin de contribuer à leur domestication. Cette étude a consisté à tester les capacités germinatives des graines de l'Ampelocissus multistriata par les procédés physiques, chimiques et mécaniques d'une part et tester l'aptitude au bouturage des tiges feuillées, des tiges feuillées avec des racines tubéreuses d'autre part. Pour cela, une surface de $400 \mathrm{~m}^{2}$ a été délimitée au sein de la Faculté des Sciences Exactes et Appliquées de l'Université de N'Djamena pour les deux tests. Par ailleurs, les procédés physiques, chimiques et mécaniques ont été menés à l'Université de Ouagadougou. Les résultats obtenus, ont montré que les tiges feuillées n'ont pas bouturé. Par contre, les tiges feuillées munies des racines tubéreuses ont bouturé à 73,23 \% . En ce qui concerne la germination des graines, tous les procédés des traitements physiques, chimiques et mécaniques n'ont pas pu lever la dormance de ces graines. Leurs téguments, très durs, n'ont pas été scarifiés et cela n'a pas permis à l'eau et l'oxygène d'y entrer pour que les graines puissent germer. Seules, les graines ayant séjourné dans le tube digestif des animaux et qui ont été scarifiées par les enzymes intestinales et les sucs digestifs ont toutes germé. Il s'agit d'une endozoochorie. La connaissance des modalités de la propagation par les graines ou par les boutures peut apporter une importante contribution à la conservation de la diversité végétale, à la domestication et à la valorisation de cette plante.

(C) 2021 International Formulae Group. All rights reserved.

Mots clés : germination, semence, clonage, bouture, Ampelocissus multistriata.

\section{Germinative capacity of seeds and aptitude for cutting of stems, tuberous roots of the species Ampelocissus multistriata (Baker) Planch.}

\begin{abstract}
This study is part of the context of valuing spontaneous plant species with nutritional and economic values in order to contribute to their domestication. This study consisted in testing the germination capacities of the seeds of Ampelocissus multistriata by physical, chemical and mechanical methods on the one hand and testing
\end{abstract}


the aptitude for cuttings of leafy stems, leafy stems with tuberous roots on the other hand. For this, an area of $400 \mathrm{~m}^{2}$ has been defined within the Faculty of Exact and Applied Sciences of the University of N'Djamena for the two tests. In addition, the physical, chemical and mechanical processes were carried out at the University of Ouagadougou. The results obtained showed that the leafy stems did not cuttings. In contrast, the leafy stems with tuberous roots cut at $73.23 \%$. Regarding seed germination, not all physical, chemical and mechanical treatment processes were able to break the dormancy of these seeds. Their very hard seed coats were not scarified, and this did not allow water and oxygen to enter them for the seeds to germinate. Only the seeds which have stayed in the digestive tract of animals and which have been scarified by intestinal enzymes and digestive juices have all germinated. It is an endozoochory. Knowing the methods of propagation by seeds or cuttings can make an important contribution to the conservation of plant diversity, to the domestication and enhancement of this plant. (C) 2021 International Formulae Group. All rights reserved.

Keywords: germination, seed, cloning, cutting, Ampelocissus multistriata.

\section{INTRODUCTION}

L'Ampelocissus multistriata est une plante spontanée, annuelle, qui, après les premières pluies de juin, pousse de la terre. Cette espèce possède des tubercules fusiformes et charnus enfoncés profondément dans le sol. C'est une plante à souche vivace tubéreuse et presque ligneuse à tiges annuelles (Nguinambaye et al., 2015a). Ampelocissus multistriata est une espèce qui se reconnait par des inflorescences munies d'une vrille. Elle se reconnait aussi par des fleurs pentamères, hermaphrodites et des fruits en baies, des graines à coupe transversale en T. C'est une plante qui se rencontre en forêt claire et en savane, sur sol sableux à sablo-argileux, sur rochers. C'est une espèce qui est largement répandue en Afrique tropicale. Aux premières rosées, des pousses herbacées sortent de terre et rampent sur le sol ou s'attachent aux herbes ou aux arbustes voisins à l'aide de leurs vrilles. Avant l'hivernage, la plante produit de gros tubercules sur lesquels poussent de longs sarments assez bien fournis. Selon l'observation des paysans, certains agents vecteurs tels que les oiseaux, les singes mangent les baies et disséminent les graines sur les plateaux, dans les rochers, le long des rivières et tout autour des termitières. Ces zones sont des stations où vivent de préférence ces plantes. Ampelocissus multistriata est une espèce qui a une diversité écologique.

De nos jours, les propriétés des composés phénoliques sont largement étudiées dans le domaine médical, où on leur reconnaît des activités antivirales, antibactériennes, anti- allergiques, anti-tumorales mais aussi antiinflammatoires (Middleton et al., 2000). En effet, les composés phénoliques sont reconnus comme de bons antioxydants par excellence (Bouayed, 2007). Nguinambaye et al. (2015b) ont montré que la teneur en polyphénols totaux des racines tubéreuses d'Ampelocissus multistriata a été de 216,946 mg EAG/g de matière sèche. En conséquence, la présence des composés phénoliques dans les extraits contribue de manière significative à leurs propriétés anti-oxydantes. Bidié et al. (2011) ont montré que les plantes qui possèdent une bonne activité antioxydante contiennent de fortes teneurs en groupement phénoliques. Des résultats similaires ont été trouvés par Tunalier et al. (2001), Fernádez-Pachón et al. (2004) et Mbaiogaou et al. (2013) qui ont montré qu'il existe une forte corrélation entre le profil en polyphénols totaux et l'activité antioxydante des extraits des plantes. Ces fortes activités antioxydantes contribueraient aux diverses activités thérapeutiques de cette plante (Syamsudin et al., 2007).

Par ailleurs, les fruits et les jeunes feuilles d'espèces Ampelocissus multistriata sont comestibles. Les tubercules sont utilisés pour l'engraissement des bœufs.

Il est donc nécessaire d'accorder un intérêt particulier à la valorisation des espèces spontanées qui constituent de ressources naturelles importantes. Mais souvent, elles sont sous- exploitées du fait de l'ignorance des valeurs nutritives de celles-ci. Ampelocissus multistriata est une espèce exploitée de façon empirique dans la zone pour les besoins 
nutritionnels et sanitaires (Mbaihougadobe, 2017). Peu d'études de caractérisation biochimique ont pu être faites pour valoriser les composés chimiques. Il est à signaler qu'en Afrique, les vertus thérapeutiques des plantes étaient connues par nos ancêtres et nos parents de façon empirique (El Rhaffari, 2002). Mais des menaces de disparition pèsent sur elles, à cause des animaux qui les broutent et les piétinent donc, il est nécessaire de rechercher sa domestication et sa pérennisation par voie germinative et par voie de bouturage. Or, la connaissance des modalités de la propagation par les graines ou par les boutures peut apporter une importante contribution à la conservation de la diversité végétale, à la domestication et à la valorisation de cette plante. En effet, les travaux portant sur la multiplication végétative ont pour but de comprendre les différents phénomènes d'adaptations développées par cette espèce pour assurer sa survie.

Ce travail a pour but de tester la capacité germinative des graines de Ampelocissus multistriata et aussi de tester sa reproduction par voie végétative.

\section{MATERIEL ET METHODES}

Une surface de $400 \mathrm{~m}^{2}$ a été délimitée au sein de la Faculté des Sciences Exactes et Appliquées de l'Université de N'Djamena et a permis la mise en place de la multiplication végétative.

La multiplication végétative est définie par Nivot en 2005 comme étant un corollaire de l'aptitude à la croissance indéfinie des végétaux. Pour cette multiplication végétative, il faut la récolte et la préparation de la bouture pour repiquage. Ainsi, les paramètres pris en compte dans le choix des parents étaient le diamètre, la hauteur, l'état sanitaire et la forme. Ainsi, les boutures étaient conditionnées sous forme de morceaux de 15 à $20 \mathrm{~cm}$ de long et de diamètre variant entre 5 et $10 \mathrm{~cm}$. Le diamètre du rhizome de jeunes pousses varie entre 15 à 25 $\mathrm{cm}$. Pour le dispositif expérimental (Figure 4), 30 pots ont été utilisés. Chaque pot était rempli de la terre argilo-sableuse.

En ce qui concerne les tests de germination, les graines sont soumises à 5 types des traitements: hydrique, chimique, mécanique, technique de vernalisation et action des enzymes digestifs.

Traitement hydrique: les graines étaient trempées dans l'eau chaude à $40^{\circ} \mathrm{C}$ durant $1 \mathrm{~h}$ et aussi dans l'eau froide à $20^{\circ} \mathrm{C}$ pendant $24 \mathrm{~h}$ (Pattanath , 1982 ; Benbada , 2013).

Traitements chimiques : les graines ont été trempées successivement dans l'eau de javel, l'acide chlorhydrique, l'acide sulfurique concentré à 96\% (Wahbi et al., 2010; Salima et al ., 2015 ).

Traitements mécaniques : la scarification se faisait premièrement à l'aide de papier abrasif (Roussel, 1992 ; Danthu et al., 1992; Ndour, 1997). Puis, ensuite, à l'aide de la loupe et d'un bistouri qui permettaient d'enlever avec délicatesse, une partie de tégument.

Technique de vernalisation: c'est une technique « en strates » qui porte le nom de stratification (Mugnier, 1979). Cette technique de vernalisation consistait à utiliser le réfrigérateur. Pour cela, trois réfrigérateurs ont été utilisés et réglés respectivement à 4,6 et 8 ${ }^{\circ} \mathrm{C}$.

Actions des enzymes digestifs: Cette technique a consisté à faire manger les fruits d'Ampelocissus multistriata par les chèvres et les graines récupérées à partir des excréments ont été semées dans des pots.

Les graines n'ayant subi aucun traitement ont été semées aussitôt. Après les traitements chimiques, les graines ont été retirées de chaque solution. Elles ont été lavées à fond dans un courant d'eau fraîche pendant 5 à $10 \mathrm{mn}$ pour éliminer toute trace de substance chimique. Elles étaient ensuite lavées à l'eau distillée (Wahbi et al, 2010). Les graines physiquement traitées sont aussitôt semées. Une partie des graines a été mise à germer dans des pots. Une autre partie des graines traitées a été déposée dans des boîtes de Pétri en plastique sur deux couches de papier filtre avec 3 répétitions par traitement, à raison d'au moins cinq (5) graines par boîte.

\section{Traitement des données}

Le logiciel photo schop a permis de traiter les images réalisées. 


\section{RESULTATS}

\section{Multiplication par voie végétative}

Cas des tiges feuillées sans rhizome

Pour les tiges feuillées sans rhizome, 3 jours après la mise en terre, elles ont commencé à sécher. Ces boutures ont complètement séché dans les différents pots de tous les trois blocs, 5 jours après.

\section{Cas des tiges feuillées avec rhizome}

Pour cet essai, les premières pousses de certains pieds ont apparu sur les tiges 5 jours après la mise en terre. Sur quinze pieds transplantés, onze ont survécu soit un pourcentage de $73,23 \%$ de réussite et un taux d'échec de 26,73\% conformément au Tableau 1.

\section{Multiplication par voie des graines}

Les traitements à l'eau froide, à l'eau tiède et à l'eau bouillie, n'ont donné aucun résultat après un mois de semis donc le taux de germination était nul. Les graines ayant subi le traitement chimique (à l'acide sulfurique, à l'eau de javel et à l'acide chlorhydrique) et celles scarifiées étaient couvertes de moisissure (Figure 8). Le taux de leur germination était nul.

Les graines ayant traversé le tube digestif des chèvres ont toutes germé (Figure 9).

Tableau 1 : Résultat de la multiplication végétative (boutures avec rhizomes).

\begin{tabular}{llll}
\hline $\begin{array}{l}\text { Blocs } \\
\text { Plants }\end{array}$ & $\mathbf{B}_{1}$ & $\mathbf{B}_{2}$ & $\mathbf{B}_{\mathbf{3}}$ \\
\hline $\mathrm{T}_{1}$ & meurt & survie & Survie \\
$\mathrm{T}_{2}$ & survie & meurt & Survie \\
$\mathrm{T}_{3}$ & survie & survie & Survie \\
$\mathrm{T}_{4}$ & meurt & survie & Survie \\
$\mathrm{T}_{5}$ & survie & survie & Meurt \\
\hline
\end{tabular}

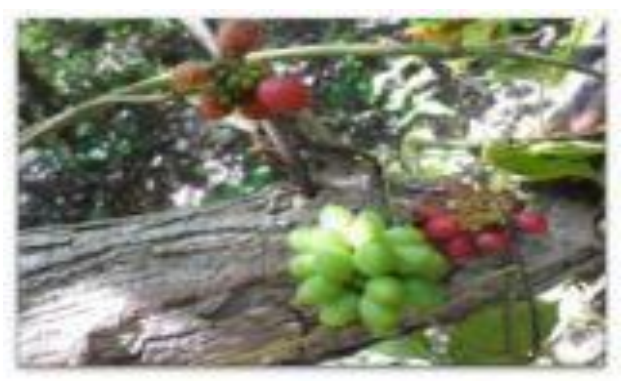

Figure 1: Espèce Ampelocissus multistriata en place dans son milieu naturel.
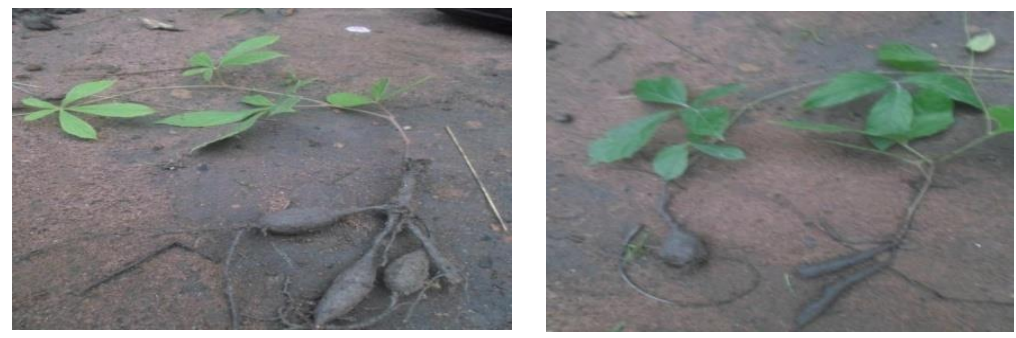

Figure 2 : des jeunes rhizomes portant des tiges feuillées pour la mise en terre. 


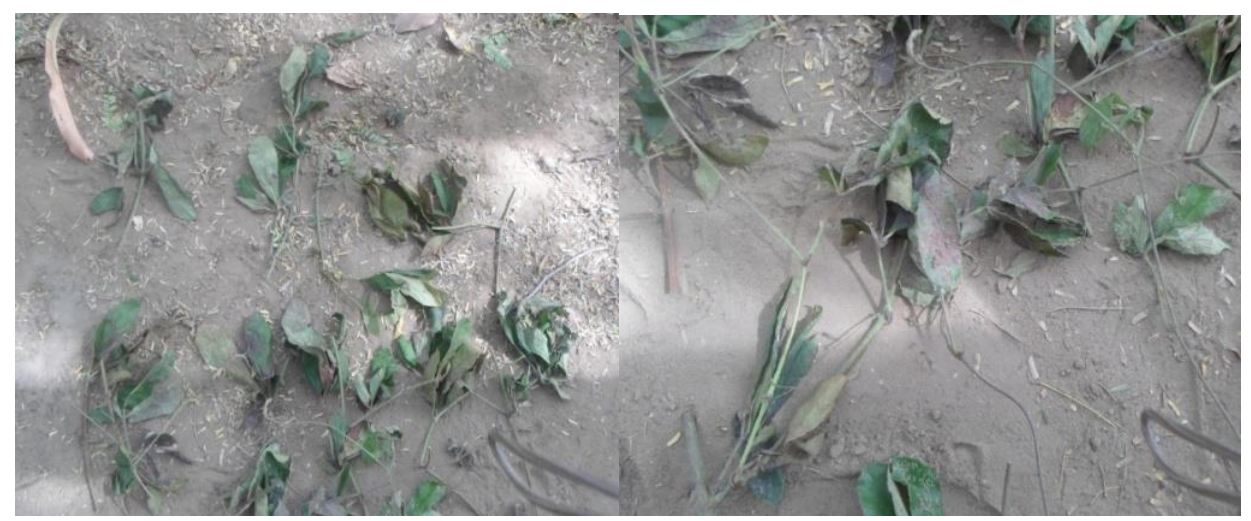

Figure 3 : Tiges feuillées sans rhizomes pour le repiquage.

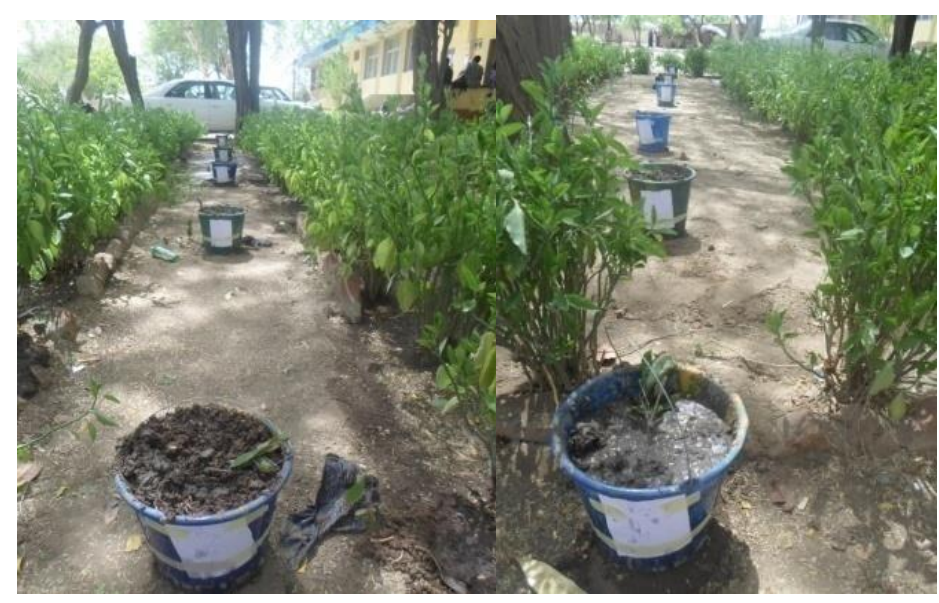

Figure 4: Dispositif expérimental de la multiplication végétative (tiges feuillées sans rhizomes et rhizomes portant des rameaux feuillés).

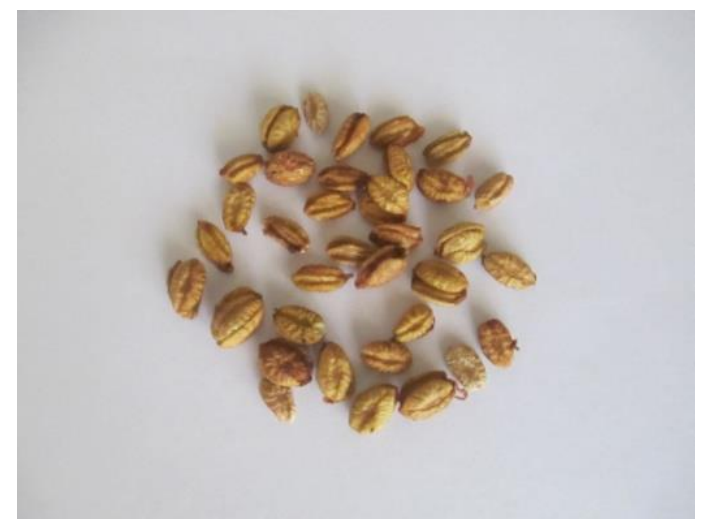

Figure 5 : Des graines saines séchées. 


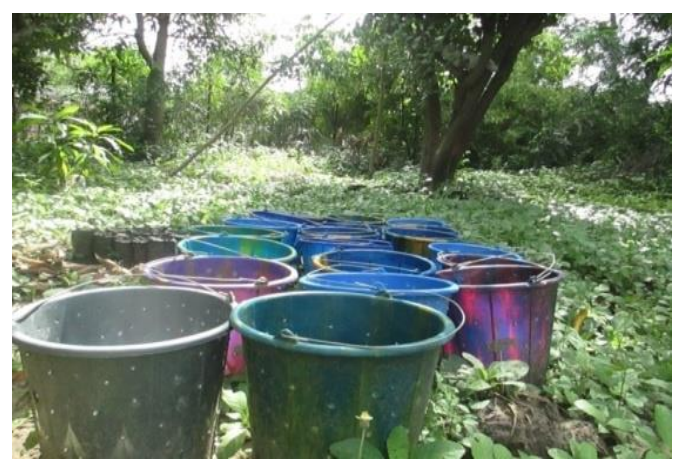

Figure 6: Dispositif expérimental semées dans des pots.

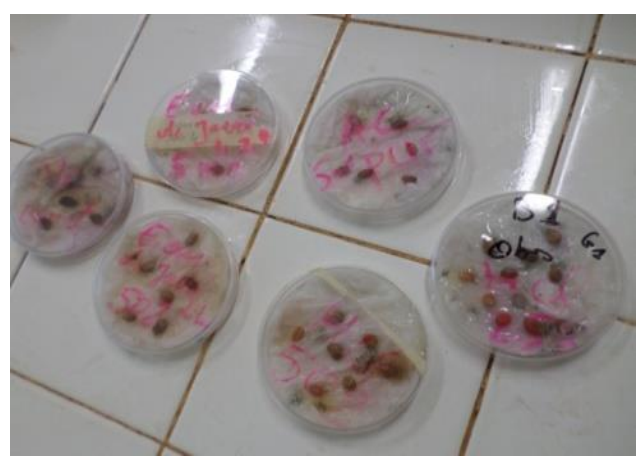

Figure 7 : Dispositif expérimental des graines semées dans des boites de pétri au laboratoire.

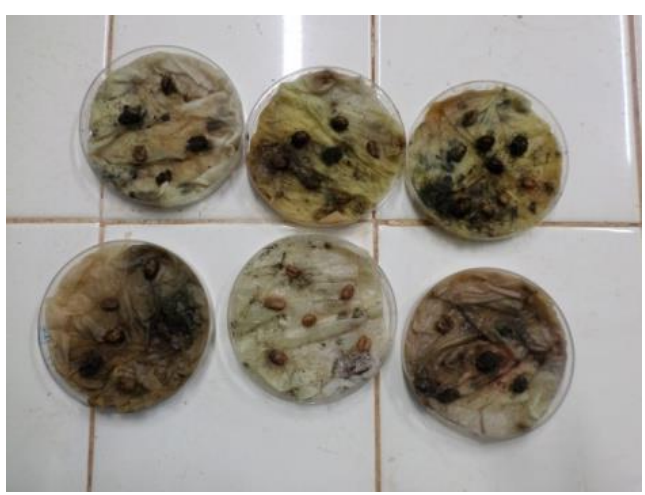

Figure 8: Graines scarifiées et celles traitées chimiquement ayant moisi.
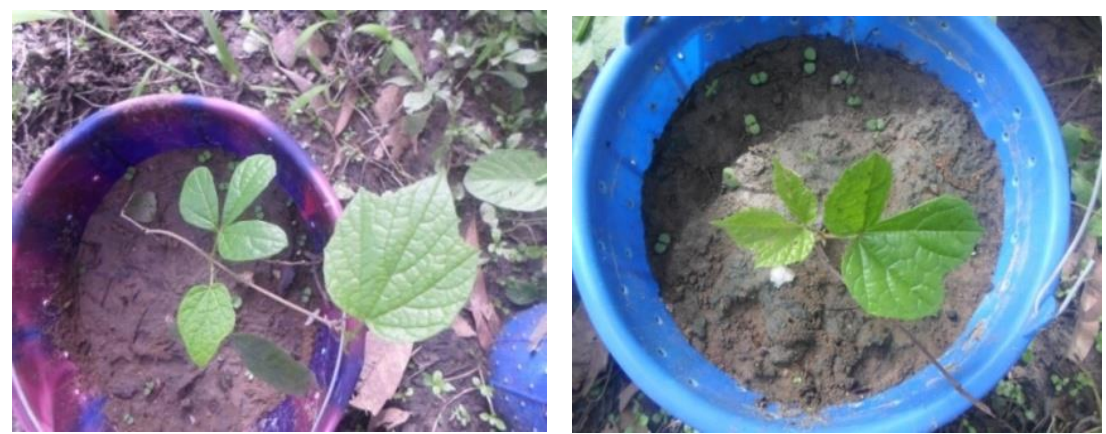

Figure 9: Tiges feuillées ayant été obtenues à partir des graines sorties des tubes digestifs des animaux. 


\section{DISCUSSION}

\section{Multiplication végétative}

Les boutures sont des fragments d'organismes, produits naturellement ou artificiellement, capables de produire un nouvel individu complet. C'est le cas chez de nombreuses plantes. Or, dans le cas des Ampelocissus multistriata, la reproduction par bouturage (tige feuillée) n'a pas été possible. Ceci pourrait s'expliquer par le fait que des cellules végétales ne seraient pas capables de reproduire un individu complet. Ainsi donc, la tige d'Ampelocissus multistriata est annuelle alors dépourvue des formations secondaires à savoir le bois secondaire et le phloème secondaire pouvant former le pachyte. Pourtant, les cellules d'un végétal sont souvent totipotentes car elles ont la possibilité de revenir à un état embryonnaire, et de se redifférencier en toute cellule spécialisée qui sera nécessaire pour former une nouvelle plante.

Pour le deuxième cas de bouturage par transplantation, le pourcentage de réussite est élevé $(73,23 \%)$. Cela serait dû aux souches de cette espèce qui est vivace donc capable de régénérer un être complet. Il faut signaler qu'avant l'hivernage, la plante produit de gros tubercules sur lesquels poussent de longs sarments assez bien fournis. C'est une plante à souche vivace tubéreuse et presque ligneuse, à tiges annuelles. C'est une plante lianoïde à souche pérenne, avec un système racinaire à tubercules allongés noirâtres (Nguinambaye, 2016).

\section{Multiplication par voie des graines}

Les graines d'Ampelocissus multistriata n'ont pas pu germer malgré les différents traitements chimiques et mécaniques qui leur sont appliqués. Ces graines qui n'ont pas pu germer ne sont pas imbibées et sont restées dures. Plusieurs possibilités peuvent expliquer cette situation. On peut noter, entre autres : les conditions des différents traitements appliqués ne seraient pas bien réunies. C'est ce qui se traduit par la présence de moisissures dans certaines boîtes de pétri. Par ailleurs, le temps que les graines ont mis pour être semées n'a pas été suffisant car certaines graines sont même réputées ne germer qu'au bout de 2-3 ans (FAO, 2012). Ce résultat est similaire aux travaux antérieurs. Ces auteurs ont trouvé que de nombreuses expériences concernant le traitement des semences font ressortir que le facteur temps est un facteur important pour l'induction d'une bonne activité germinative (Mbaye, 2002 ; Kheloufi, 2017). En plus, l'eau et l'oxygène n'auraient pas pu atteindre l'embryon à cause de l'imperméabilité des téguments. Or, Soltner (2007) a montré que la graine exige la réunion des conditions favorables à savoir l'eau, l'oxygène et la température pour germer. Les divers traitements appliqués auraient pu lever la dormance. Ce qui n'a pas été le cas. Pour cela, on peut envisager que cette inhibition est d'origine embryonnaire. Les résultats obtenus révèlent que les différents traitements chimique et mécanique appliqués aux graines d'Ampelocissus multistriata ne les ont pas permis de germer. Ceci peut s'expliquer par le fait que la dormance des graines est d'origine tégumentaire. La rigidité des téguments ne permet pas l'eau et l'oxygène d'atteindre l'embryon. En outre, Finkelstein et son équipe en 2008 ont montré que la dormance des graines fournit un mécanisme permettant aux plantes de retarder la germination jusqu'à ce que les conditions soient optimales pour la survie de la prochaine génération.

Certaines plantes possèdent des fruits pulpeux autour des graines. Les animaux, attirés par la valeur nutritionnelle des fruits, les mangent et, par la même occasion, ingèrent les graines. Les graines, contrairement à la chair des fruits, ne seront pas digérés et achèveront leur voyage à travers le tube digestif de l'animal. Or, les graines ayant transité par le tube digestif ont germé simplement par ce que les enzymes intestinales et les sucs digestifs ont pu scarifier les téguments des graines pour leur permettre de germer. L'élimination par les sucs digestifs d'inhibiteurs chimiques internes pourrait lever la dormance dans certains cas. Cette reproduction ne peut se produire que si les conditions stationnelles sont réunies. Et aussi, il faut un rapprochement entre la morphologie des graines (capables de résister à la digestion) et celle des oiseaux (ne détruisant 
pas les graines qu'ils ingèrent). Si les graines ont germé, cela est dû à une scarification biochimique. Les graines, dans les tubes digestifs des oiseaux, chèvres ou singes seraient scarifiées par des enzymes et auraient rendu souples les téguments. Ce qui permettrait à l'oxygène et l'eau d'atteindre facilement l'embryon. Ce que corroborent les travaux de (Dabonneville, 2018). Cet auteur explique que le séjour à l'intérieur d'un tube digestif joue parfois un rôle majeur dans le processus de germination des graines. En effet, certaines graines sont entourées d'une enveloppe protectrice, le tégument, très épaisse dont la dureté et l'imperméabilité empêchent la germination (car interdisant tout échange d'oxygène et d'eau avec l'extérieur). En traversant le système digestif, la graine est en contact avec les sucs digestifs qui attaquent ce tégument et réduisent son épaisseur et sa dureté. Ainsi, les embryons des graines doivent rester protégés derrière la barrière physique que constituent les téguments résistant à toute forte attaque abrasive mécanique ou chimique. Cependant, si l'enveloppe n'est pas totalement imperméable, des facteurs chimiques internes peuvent intervenir. Selon la grosseur des graines, leur dureté et la résistance de leur enveloppe, 10 à $40 \%$ étaient retrouvées dans les fèces 1 à 10 jours après ingestion. Beaucoup de ces graines ont subi une baisse de leurs facultés germinatives, d'autant plus accentuée que leur séjour dans le tractus digestif fut plus long (Chasseriaud, 2018).

Certaines plantes ont recours aux animaux pour disperser leurs graines. C'est ce qu'on appelle la zoochorie. La zoochorie peut être passive, c'est-à-dire que les animaux transportent les graines involontairement. C'est le cas de l'épizoochorie, lorsque des animaux transportent des graines qui se sont fixées à leur pelage ou à leur plumage. La zoochorie peut aussi être active, quand les animaux participent à la dissémination des graines de par leurs actions. C'est le cas de l'endozoochorie, qui correspond à une dissémination des graines via le tube digestif des animaux. Cela se passe lorsque les animaux ingèrent les graines qui vont alors transiter par leur tube digestif et terminer leur parcours là où l'animal soulagera ses entrailles (Chasseriaud, 2018). Beaucoup d'auteurs ont ainsi montré le rôle considérable que pouvait jouer le bétail dans la dispersion des mauvaises herbes. Il a par ailleurs été montré que, pour les plantes se disséminant par endozoochorie, les graines issues d'excréments se développent plus que les graines simplement tombées de la plante-mère (Chasseriaud, 2018). Par contre,

Niang-Diop et al. (2010) ont montré que le passage des graines de Prosopis africana dans le tractus digestif des animaux ne semble pas améliorer leur capacité de germination. Ce passage des graines dans le tractus digestif des bœufs n'améliore pas la germination des graines de l'espèce. Un résultat similaire a été obtenu par Danthu et al. (1996) après le passage des graines dans le tractus digestif des ruminants. Cette forme de prédation des graines, qui dépend fortement des espèces, constitue parfois un facteur positif à la germination des espèces (Tybirk et al., 1994).

\section{Conclusion}

L'étude menée révèle que l'Ampelocissus multistriata a la capacité de se régénérer aussi bien par la voie germinative que la voie végétative. La connaissance des modalités de la reproduction par les fruits ou les graines et par les boutures peut apporter une importante contribution à la conservation de la diversité végétale et à la valorisation des plantes. Notre étude a révélé que les cellules des tiges sans racine ne peuvent pas régénérer un nouvel individu. Par ailleurs, la multiplication des tiges avec racine a donné un pourcentage élevé. Leur taux de multiplication est de 73,23\%. D'autre part, les graines d'Ampelocissus multistriata, malgré les différents traitements appliqués, n'ont pas pu germer. Pourtant, par scarification biochimique, grâce aux enzymes intestinales et aux sucs digestifs les oiseaux, les singes, les chèvres parviennent à faire germer ces graines indépendamment des humains.

\section{CONFLITS D'INTERETS}

Ce document ne souffre d'aucun conflit d'intérêts. 


\section{CONTRIBUTIONS DES AUTEURS}

Les deux auteurs ont tour à tour fait la relecture du manuscrit en faisant des amendements. Ils ont participé financièrement à la réalisation de travaux cet article.

\section{REMERCIEMENTS}

Je tiens à remercier les co-auteurs qui ont lu et corrigé le manuscrit. Je remercie l'Université de Ndjamena à travers la faculté des Sciences Exactes et Appliquées qui a soutenu matériellement et financièrement les travaux de cette étude. Je tiens à remercier vivement l'Université d'accueil, l'Université de Ouagadougou dont le soutien n'a pas été moindre.

\section{REFERENCES}

Benbada S. 2013. Amélioration du taux de germination des grains d'Acacia reddiana pour lever leur inhibition tégumentaire, Universite Kasdi Merbah-ouargla Mémoire $50 \mathrm{P}$.

Bidié P, Banga B, Nguessan AH, Adou F, Yapo JD, Djaman AJ. 2011. Activités ant- oxydantes de dix plantes médicinales de la Pharmacopée ivoirienne. Sci. Nat., 8: 1-11. DOI: 10.4236/ns.2021.139030.

Bouayed J. 2007. Étude de la corrélation anxiété/statut oxydatif des granulocytes chez la souris et évaluation des effets antioxydants/neuroactifs des polyphénols extraits de Prunus domestica L. Thèse de doctorat, Université Verlaine-Metz, p.368.

Chasseriaud M. 2018. Chronique rédigée et présentée par Océane Bartholomée pour le Mag DSciences - Chronique ré-éditée pour Echosciences par Chasseriaud Mathilde. http://www.conservationnature.fr/article1.php.

Dabonneville C. 2018. Des fruits pulpeux pour disséminer les graines. Espèces - Revue d'Histoire naturelle, 27 : 69-73. DOI : 10.3406/rhs.

Danthu P, Roussel, Dia M, Sarr A. 1992. Effect of pretreatement on the germination of Acacia senegal seeds. Seed Sci.Technol., 20: 111117. https://doi.org/10.15258/sst.2019.4 7.1.13
Danthu P, Ickowicz A, Friot D, Manga D, Sarr A. 1996. Effet du passage par le tractus digestif des ruminants domestiques sur la germination des graines de légumineuses ligneuses des zones tropicales sèches. Revue Elev. Med. Vét. Pays Trop., 49(3): 235-242.

DOI : 10.1051/PARASITE/2014002.

El Rhaffari 1, Zaid A. 2005. Pratique de la phytothérapie dans le sud-est du Maroc (Tafilalet). Un savoir empirique pour une pharmacopée rénovée. IRD Éditions, 2002. Terms of use: http://www.openedition.org/6540.

FAO : 2012.Guide de manipulation des semences. http://www.greffer.net/?p=194

Fernádez-Pachón MS, Vilaňo, Garcia-Parilla MC, Troncoso AM. 2004. Antioxydant activity of wines in relation with their phenolic composition. Analytica Chimica Acta $\quad \mathbf{5 1 3}:$ 113-118. https://doi.org/10.1016/j.aca.2021.33902 4.

Finkelstein, R., Reeves W., Ariizumi T., Steber C. 2008. Molecular aspects of seed dormancy. Ann Rev Plant Biol. 59 : 387415

DOI:

10.1146/annrev.arplant.59.032607.09274 0.

Kheloufi A, Mansour LM. 2017. Effet de l'acide sulfurique sur la germination d'un arbre fourrager Acacia nilotica (L.) subsp tomentosa . Livestock Research for Rural Development, 29 (2). Retrieved October 9, 2021, from http://www.lrrd.org/lrrd29/2/khel29027. html

Mbaihougadobe S. 2017. Phytochimie et pharmacologie des plantes utilisées dans le traitement de la goutte au Tchad. Thèse de doctorat unique, Université Marien N'Gouabi, République du Congo p.173.

Mugnier C. 1979. Caractéristiques de la vernalisation d'une plante vivace, le Cresson de terre, Barbaraea praecox $\mathrm{Br}$. R. Bulletin de la Société Botanique de France. Lettres Botaniques, 126(3): 279-292. DOI: 10.1080/01811797.1979.10824398

Mbaiogaou A, Ouédraogo M, Palé E, Naitormbaide M, Mahamout Y, Nacro M. 2013. Étude comparative des teneurs en 
polyphénols et en antioxydants totaux d'extraits de graines de 44 variétés de Voandzou (vigna subterranea (L.) Verdcourt). Int. J. Biol. Chem. Sci., 7(2) : 861-871. DOI: 10.4314/ijbcs.v7i2.41 .

Mbaye N, Diop AT, Guèye M, Diallo AT, Sall CE, Samb PI. 2002. Etude du comportement germinatif et essais de levée de l'inhibition tégumentaire des graines de Zornia glochidiata Reichb. ex DC., légumineuse fourragère. Revue Elev. Ed. Vet. Pays Trop., 55 (1) : 47-52. DOI: 10.1051/PARASITE/2014002

Middleton E, Kandaswami C, Theoharidies TC. 2000. The effects of plant flavonoids on mammalian cells: implications for inflammation, heart disease and cancer. Pharmacological Reviews, 52: 673751. DOI: http://dx. doi.org/10.1136/bmjopen2017-015860.

Ndour P. 1997. Comportement de quelques espèces du genre Acacia en condition de stress hydrique et salin simulé. DEA en biologie Végétale, Univ. CH. Anta Diop, Dakar (Senegal), p.80 .

Nguinambaye MM, Rasmata N, Mbayngone E, Djinet AI, Badoua B, Zoumbiéssé T. 2015a. Distribution et usages des Ampelocissus dans la zone de Donia au sud du Tchad. Int. J. Biol. Chem. Sci. 9(1): $186-199 . \quad$ DOI: 10.4314/ijbcs.v9i1.17.

Nguinambaye MM, Rasmata N, Savadogo A, Djinet IA, Zoumbiéssé T. 2015b. Composés organiques et activités antioxydantes de Ampelocissus multistriata du Tchad. Journal of Applied Biosciences, 91: 8970-8479. DOI : http://dx.doi.org/10.4314/jab.v91i1.7

Nguinambaye MM. 2016. Étude écophysiologique et ethnobotanique d'Ampelocissus multistriata (Baker) Planch. (Vitacées Ampélidacées) dans la Sous-préfecture de Donia située au sud du Tchad. Thèse de doctorat Unique, Burkina Faso p. 106.
Niang-Diop F, Sambou B, Lykke AM. 2010. Contraintes de régénération naturelle de Prosopis africana : facteurs affectant la germination des graines. Int. J. Biol. Chem. Sci., 4(5): 1693-1705. DOI: 10.4314/ijbcs.v4i5.65578.

Nivot N. 2005. Essais de germination et de bouturage de six espèces indigènes sciaphytes du canada. Mémoire p.117.

Pattanath 1982. Guide de manipulation des semences forestières. DOI : http://www.fao.org/3/ad232f/ad232f11.h tm

Salima Kebbas, Zahra-Wassila Lekehal, Fatiha Aid. 2015. Analyze of Different Methods of Scarification and Biochemical Composition of Acacia Tortilis Subsp Raddiana Seeds. Bulletin USAMV series Agriculture, 72(1): 49-54. DOI : 10.15835/arspa.v93i1-2.11251

Soltner D. 2007. Les Bases de la Production Végétale. Tome III. La Plante. Ed. Collection Sciences et Techniques Agricoles : Paris ; 304p.

Syamsudin SK, Broto S. 2007. Screening of some extracts from Garcinia parvifolia Miq (Gouttiferae) for antiplasmodial, antioxydant, cytotoxic and antibacterial activities. Asian J. Plant Sci, 6: 972-976. DOI: 10.3923/ajps.2007.972.976

Tunalier Z, Kosar M, Ozturk N, Baser KHC, Duman N, Kirimer N. 2001. Antioxidant properties and phenolic composition of Sidirits species. Chemistry of Natural Compounds, 40: 206-210. DOI: https://doi.org/10.1007/s10600-0089073-4.

Tybirk K, Schmidt LH, Hauser T. 1994. Notes on soil seed banks of African Acacias. Afr. J. Ecol., 32: 327-330. DOI: 10.1111/j.1469.

Wahbi J, Lamina H, Naoufel S, Mohamed L. 2010. Étude de la germination des graines d'Acacia tortilis sous différentes contraintes abiotiques. Biotechn. Agron. Soc. Environ., 14(4): 643-652. https://popups.uliege.be/17804507/index.php?id=6419. 